\title{
When psychiatric patients interact with computer terminals: Problems and solutions
}

\author{
EARL B. COLE, JAMES H. JOHNSON, and THOMAS A. WILLIAMS \\ Systems and Evaluation Unit, Mental Health and Behavioral Sciences Service \\ Veterans Administration Hospital, Salt Lake City, Utah 84113 \\ and \\ Department of Psychiatry, University of Utah College of Medicine, Salt Lake City, Utah 84112
}

\begin{abstract}
A computer system for on-line psychiatric patient assessment has been developed at the Veterans Administration Hospital in Salt Lake City. Unlike the typical on-line computer system designed for skilled terminal operators, this system is designed to interact with unskilled psychiatric patients. Special design considerations necessary to support this approach are discussed.
\end{abstract}

On-line interactive computer systems are typically designed and developed for use in controlled settings where users have requisite experience and skills. Unlike typical on-line systems, the computer system developed at the VA Hospital in Salt Lake City has been designed to interact with psychiatric patients who are unskilled in computer operation. This system uses interactive computer terminals to administer psychological assessment instruments directly to psychiatric patients as an integral part of the admissions procedure of the prototype Psychiatric Assessment Unit (PAU) (Johnson, Giannetti, \& Williams, 1975; Johnson \& Williams, 1975; Williams, Johnson, \& Bliss, 1975). The design for this system required special considerations to support terminal interaction and use by unskilled psychiatric patients. A previous report has detailed the general developmental requirements of this system (Cole, Johnson, Williams, 1975). The purpose of this paper is to describe the patient-terminal interface characteristics of the PAU assessment system. Specific problems encountered in design, and in our experiences with over 2,000 patients, are discussed. These considerations are presented as aids to others considering the development of similar systems.

\section{DESIGN CONSIDERATIONS}

The basic objectives of an on-line psychiatric

This project has been supported in part by contracts from the Health Service, Veterans Administration Central Office in Washington, D.C. The authors recognize the Salt Lake City VA Hospital PAU staff for their contributions to this project and extend thanks to Annette Kearl and Susan Sudbury for their assistance in preparation of this manuscript. Requests for reprints should be addressed to Earl B. Cole, Systems and Evaluation Unit, VA Hospital, Salt Lake City, Utah 84113. assessment system are to present self-report psychological questionnaires directly to psychiatric patients through interactive computer terminals, and to collect meaningful responses even though patients are likely to be in emotional distress at the time of testing and naive about computer operation. To meet these objectives, systems design must address the needs of people in distress, as well as the training needs of patients who are unskilled in computer operations. In addition, system design must insure that valid data are gathered. It must also minimize the effect of computer systems failures upon patients using the system.

\section{Patient Needs}

A complete and comprehensive workup of a psychiatric patient requires a lengthy assessment battery. Concentration during the entire testing process is essential to insure maximum validity, but it is extremely difficult to achieve with persons in emotional distress. Interruption of testing at reasonable intervals is necessary to help the patient maintain the needed concentration. Merely leaving a terminal unattended during a patient's absence makes it vulnerable to disturbance and unavailable for testing other patients. This problem is resolved in the PAU system through the use of "continuation" and "status monitoring" functions. A staff member may suspend testing at any point in the assessment process by requesting the system to discontinue the patient and free the terminal for use. When testing is suspended, the assessment status of the patient is recorded by the system. On the patient's return, the status indicator is used to resume testing at the point where it was discontinued.

A patient's concentration may also be interrupted if he is distracted by lengthy delays between questions. The system must make use of priority 
scheduling schemes to insure minimum response time on patient terminals. Other processes that may be operating in conjunction with patient testing must be handled by the system on a free and available resource basis. Priority scheduling also reduces the overall testing time, which is another essential goal of an on-line system.

These features permit needed flexibility in patient testing and permit maximum utilization of valuable terminal equipment.

\section{TRAINING}

In order to minimize training requirements, terminals used for patient assessment must be simple in operation, easy to read, and not overly distracting to the patient. An ideal terminal would have a few well-spaced keys. large characters, and simple appearance. Unfortunately, available terminal devices are generalized in design. The confusion generated by a complex keyboard can be reduced by keyboard covers which expose only those keys necessary for testing. This is strongly recommended in systems which use single-response questionnaires, exclusively. However, if items requiring free-form responses to an item are used, the entire alphanumeric portion of the keyboard must be available. In this case, one can resort to special marks or colors on frequently used keys. Further simplification can be produced by using uniform response formats. Available questionnaires may require "true-false," alphabetic, or numeric responses to items. Standardization of the response format within and between questionnaires contributes greatly to simplicity of operation and facilitates patient training. Furthermore, presenting easily comprehensible instructions directly on the CRT, and allowing the patient to try a few sample test items prior to each instrument, reduces erroneous data. This design, along with supervision by a staff member, will give the patient the basic skills necessary to interact successfully with the computer terminals.

\section{VALIDITY}

Simplified operation and comprehensive instructions can aid in solving the problem of mechanical operation of computer terminals, but attention must also be given to curious, malicious, and careless use of the terminal equipment. The unsupervised setting necessary for the efficient self-report patient assessment requires special system design to monitor patient responses and to insure useful and valid information.

Monitoring of patient responses is accomplished in the PAU system through setting response limits on items. Each item presented to the patient has an associated set of limits within which all acceptable responses will fall. Each response is compared against these limits; the system then repeats the question if the limits are exceeded. In cases of misunderstanding of operating instructions or accidental mis-entry of data, the patient is permitted to re-answer when the question is repeated. However, consecutive erroneous responses will result in a message being presented to the patient directing him to request assistance from a staff member. All further responses will be ignored by the system until the staff member permits testing to be resumed. Additional provision is made for errors detected by the patient himself. Mis-entry or typing errors are corrected through depressing the "rub out" key on the keyboard. This removes the incorrect character from the screen and permits the entry of the desired response.

Although the system design includes means for eliminating totally erroneous responses, validity of information must also be considered. This problem is complex and cannot be monitored simply by examining individual responses. It must be approached in an empirical manner. The solution developed for the PAU system involves the administration of a brief response validity questionnaire at the beginning of the assessment process. This procedure identifies patients who are deliberately malingering, or those who are too psychotic to provide usable information. If the results of this questionnaire indicate valid responses, the assessment process is continued. Otherwise, an attempt is made to test the patient at a later date. Response monitoring and initial validity screening, plus the validity scales of standard psychological instruments such as the MMPI, aid in insuring that valid and useful clinical data will be derived from the assessment process.

\section{COMPUTER FAILURES}

Computer reliability is a major concern in any system, but it is even more critical in a patient assessment system. Unexpected system failures encountered during the 1st year of actual operation of PAU demonstrated the need for an additional design effort to minimize the effect of these failures. A patient who has nearly completed a lengthy assessment questionnaire becomes understandably frustrated when the questionnaire has to be completely readministered because data are lost as a result of some system failure. An error restart feature, which continually monitors system information and patient testing status, was implemented at PAU to solve this problem. At frequent intervals, status information and test data 
are recorded on mass storage devices which are less susceptible to system failures. In the advent of a systems failure, this information remains available to restart testing when the systems problem has been corrected. This scheme for error restart results in little or no loss of test data. In fact, system interruption often goes unnoticed by patients being tested.

\section{DISCUSSION}

A frequently asked question about on-line interactive assessment is, "Is it practical?" The answer to this question is that it can be made practical. Terminals must be simple to use, and systems safeguards must be added in order to insure reliable systems operation and the extraction of valid information. If sufficient attention is given to the needs of the patients, and to the demands of the setting in which they must interact with the system, practicality is assured.

At the Utah project, we have attempted to design a system which would facilitate the collection of comprehensive evaluation data prior to intake triage decision making. Design strategies involved in such a system are multifaceted in that administrative, clinical care, and patient needs must all be considered. In this paper, we have attempted to document design considerations aimed solely at developing a system which is usable by patients. Unless patients are able to interact easily with such a system, all other considerations are for naught.

\section{REFERENCES}

Cole, E. B., Johnson, J. H., \& Williams, T. A. Design considerations for an on-line computer system for automated psychiatric assessment. Behavior Research Methods \& Instrumentation, 1975, 7, 195-198.

Johnson. J. H., Giannetti, R. A., \& Williams, T. A. Realtime psychological assessment and evaluation of psychiatric patients. Behavior Research Methods \& Instrumentation, 1975. 7. 199-200.

Johnson, J. H., \& Williams, T. A. The use of on-line computer technology in a mental health admitting system. American Psychologist, 1975, 30, 388-390.

Williams, T. A., Johnson, J. H., \& Bliss, E. L. A computerassisted psychiatric assessment unit. American Journal of Psychiatry, 1975, 132. 1074-1076. 